\title{
Effects of Mo, Nb, Ta, Ti, and Zr on Mechanical Properties of Equiatomic Hf-Mo-Nb-Ta-Ti-Zr Alloys
}

\author{
Ko-Kai Tseng ${ }^{1}$, Chien-Chang Juan ${ }^{1}$, Shuen Tso ${ }^{2}$, Hsuan-Chu Chen ${ }^{2}$, Che-Wei Tsai ${ }^{1,2}$ (I) and \\ Jien-Wei Yeh ${ }^{1,2, *}$ \\ 1 Department of Materials Science and Engineering, National Tsing Hua University, Hsinchu 30013, Taiwan; \\ zouts2@gmail.com (K.-K.T.); d943554@oz.nthu.edu.tw (C.-C.J.); chewei@mx.nthu.edu.tw (C.-W.T.) \\ 2 High Entropy Materials Center, National Tsing Hua University, Hsinchu 30013, Taiwan; \\ a8701787017@gmail.com (S.T.); xiphofly@gmail.com (H.-C.C.) \\ * Correspondence: jwyeh@mx.nthu.edu.tw; Tel.: +886-3-5719558
}

Received: 30 November 2018; Accepted: 21 December 2018; Published: 25 December 2018

\begin{abstract}
Nowadays refractory high-entropy alloys (RHEAs) are regarded as great candidates for the replacement of superalloys at high temperature. To design a RHEA, one must understand the pros and cons of every refractory element. However, the elemental effect on mechanical properties remains unclear. In this study, the subtraction method was applied on equiatomic HfMoNbTaTiZr alloys to discover the role of each element, and, thus, HfMoNbTaTiZr, HfNbTaTiZr, HfMoTaTiZr, HfMoNbTiZr, HfMoNbTaZr, and HfMoNbTaTi were fabricated and analyzed. The microstructure and mechanical properties of each alloy at the as-cast state were examined. The solid solution phase formation rule and the solution strengthening effect are also discussed. Finally, the mechanism of how $\mathrm{Mo}, \mathrm{Nb}, \mathrm{Ta}, \mathrm{Ti}$, and $\mathrm{Zr}$ affect the HfMoNbTaTiZr alloys was established after comparing the properties of these alloys.
\end{abstract}

Keywords: high-entropy alloys; refractory high-entropy alloys; alloys design; elevated-temperature yield strength; solid solution strengthening effect

\section{Introduction}

Refractory elements, including Rhenium (Re), Molybdenum (Mo), Niobium (Nb), Tantalum (Ta), and Tungsten (W) [1] are very important for improving mechanical properties in advanced alloys such as Titanium alloys and Nickel-base superalloys. Generally, elements with a melting point higher than Titanium (Ti), such as Chromium (Cr), Hafnium (Hf), Osmium (Os), Ruthenium $(\mathrm{Ru})$, Vanadium $(\mathrm{V})$, and Zirconium $(\mathrm{Zr})$ are also classified as refractory elements. In Titanium alloys, refractory elements, especially Mo and $\mathrm{W}$, are all beta stabilizers and possess a strong solid solution strengthening characteristic [2]. $\mathrm{Nb}$ forms $\mathrm{Ni}_{3} \mathrm{Nb} \gamma$ phase in Nickel-base superalloys such as Inconel 718 [3]. The improved creep resistance of the sixth generation superalloy TMS-238 mainly results from Re and Ru additions [4]. Refractory alloys also play significant roles in industry. In the second generation nuclear power plants, the most-used materials for fuel cladding are Zircoloy 2 and Zircoloy 4. The Niobium alloy C103 is used for the nozzle extension of satellites [5]. Refractory alloys are also known as having the great potential for elevated-temperature applications because of their high strength at elevated temperature. According to thermodynamics, thermal efficiency of a turbine engine could be enhanced by increasing the turbine inlet temperature. However, the melting point of Nickel-base superalloy limits the application itself above $1200^{\circ} \mathrm{C}$. Therefore, it is necessary to develop new refractory alloys, especially for applications at temperatures higher than $1200^{\circ} \mathrm{C}$.

In 2004, Professor Yeh and his group published the concept of high-entropy alloys (HEAs) [6]. He defined high-entropy alloys (HEAs) as having five or more major elements beneath 5-35 at.\%, 
and minor elements below five at.\%. In 2006, he also established four core effects of HEAs [7]: High-entropy, severe-lattices-distortion, sluggish-diffusion, and the cocktail effect. With the new concepts, scientists are able to develop and expand alloys without restrictions [8-12]. Some HEAs have been found to have attractive properties on diffusion [13], oxidation [14], corrosion [15], fatigue [16], creep [17], fracture toughness [18], and elevated-temperature strength [19]. High-entropy superalloys (HESAs) [20], eutectic HEAs (EHEAs) [21], light-weight HEAs (LWHEAs) [22], refractory high-entropy alloys (RHEAs) [23], etc. have been proposed and attract increased attention. In 2010, Senkov and Miracle first published RHEAs, MoNbTaW and MoNbTaVW $[23,24]$. These two alloys possess body-centered cubic (BCC) structure and have excellent elevated-temperature yield strength which is around $400 \mathrm{MPa}$ at $1600{ }^{\circ} \mathrm{C}$. But their density is much higher, and the room temperature compressive ductility is very low. In 2012, they published the equiatomic composition of HfNbTaTiZr, which possesses excellent compressive ductility up to $50 \%$, lower density but poor elevated-temperature yield strength [25,26]. From then on, there were over 150 papers published concerning RHEAs [19].

There are some researches working on the addition of Al [27,28], Mo [29], Ti [30], V [31], or $\mathrm{Zr}$ [32], but few of them focus on the elevated-temperature mechanical performance and the overall effect of the constituent elements. To understand the elemental effect on mechanical properties, equiatomic HfMoNbTaTiZr alloy is firstly designed as a base alloy by adding the high modulus refractory element Mo to HfNbTaTiZr. Then the subtraction method is used to analyze each elemental effect in the equiatomic HfMoNbTaTiZr. Thus, in this study, six alloys HfMoNbTaTiZr, HfNbTaTiZr, HfMoTaTiZr, HfMoNbTiZr, HfMoNbTaZr, and HfMoNbTaTi are investigated and compared on their microstructure. Further, the compressive properties at room temperature and at elevated temperature are investigated. By comparing HfMoNbTaTiZr and HfNbTaTiZr, the influence of the addition of Mo can be understood. Likewise, the influence of the addition of $\mathrm{Nb}, \mathrm{Ta}$, $\mathrm{Ti}$, or $\mathrm{Zr}$ can be articulated. In addition, promising compositions were found and further improved design is suggested. The solid solution phase formation rule and the solid solution strengthening effect will be discussed.

\section{Materials and Methods}

The experimental Hf-Mo-Nb-Ta-Ti-Zr alloy series was prepared by vacuum-arc melting. The purity of raw elements including $\mathrm{Hf}, \mathrm{Mo}, \mathrm{Nb}, \mathrm{Ta}$, and $\mathrm{Zr}$ was $99.9 \mathrm{wt} . \%$, and that of Ti was $99.99 \mathrm{wt} . \%$. The melting points of each constituent element used in alloys are shown in Table 1. These pure metals were stacked together in the sequence of low melting point to high melting point from bottom to top. The stacked metals were melted together in a water-cool copper mold and solidified therein. The ingot of each alloy was flipped and re-melted, at least, four times to improve the chemical homogeneity. The crystal structure of the alloy samples taken from the portion near the copper mold was examined with the Shimadzu XRD-6000 X-ray diffractometer (SHIMADZU CORPORATION, Kyoto, Japan), operated at $30 \mathrm{kV}$ and $20 \mathrm{~mA}$ with a scanning rate of $4^{\circ} / \mathrm{min}$ from $20^{\circ}$ to $100^{\circ}$. JEOL JSM-5410 (JEOL Ltd., Tokyo, Japan) scanning electron microscope (SEM) and JXA-8500F FE-EPMA (JEOL Ltd., Tokyo, Japan) was used to analyze the samples in backscattering electron (BSE) mode. Energy dispersive spectrometry (EDS) was also used to confirm the chemical compositions. The cylindrical samples for the compression test were $3.6 \mathrm{~mm}$ in diameter and $6 \mathrm{~mm}$ in height. The room temperature compression tests were conducted with Instron 4468 (INSTRON, Norwood, MA, USA) universal testing machine, and the high temperature compression tests were performed on Gleeble-3500 (DYNAMIC SYSTEMS INC, Poestenkill, NY, USA) thermal-mechanical simulator. All tests were examined at the crosshead speed of $0.36 \mathrm{~mm} / \mathrm{min}$, which imposed the strain rate of $10^{-3} \mathrm{~s}^{-1}$ on the samples. 
Table 1. Results of scanning electron microscope-energy dispersive spectrometry (SEM-EDS) analysis (at. \%). Nominal composition means the designed composition. DR means the dendritic region. ID means the interdendritic region.

\begin{tabular}{cccccccc}
\hline & Element & Hf & Mo & Nb & Ta & Ti & Zr \\
\hline \multirow{3}{*}{ HfMoNbTaTiZr } & Nominal & 16.7 & 16.7 & 16.7 & 16.7 & 16.7 & 16.7 \\
& DR & 14.3 & 18.4 & 19.5 & 24.4 & 12.4 & 10.8 \\
& ID & 21.1 & 13.6 & 12.3 & 9.9 & 18.3 & 24.7 \\
\hline \multirow{3}{*}{ HfNbTaTiZr } & Nominal & 20.0 & - & 20.0 & 20.0 & 20.0 & 20.0 \\
& DR & 18.5 & - & 22.4 & 27.4 & 18.2 & 13.5 \\
& ID & 22.6 & - & 17.5 & 12.8 & 20.2 & 26.9 \\
\hline \multirow{3}{*}{ HfMoTaTiZr } & Nominal & 20.0 & 20.0 & - & 20.0 & 20.0 & 20.0 \\
& DR & 20.6 & 21.4 & - & 23.9 & 18.3 & 15.7 \\
& ID & 24.4 & 16.2 & - & 11.0 & 21.2 & 27.1 \\
\hline \multirow{2}{*}{ HfMoNbTiZr } & Nominal & 20.0 & 20.0 & 20.0 & - & 20.0 & 20.0 \\
& Overall & 20.8 & 20.6 & 19.7 & - & 19.2 & 19.7 \\
\hline \multirow{2}{*}{ HfMoNbTaZr } & Nominal & 20.0 & 20.0 & 20.0 & 20.0 & - & 20.0 \\
& DR & 18.5 & 20.8 & 21.7 & 24.5 & - & 14.5 \\
& ID & 27.0 & 15.6 & 13.5 & 9.9 & - & 34.0 \\
\hline \multirow{2}{*}{ HfMoNbTaTi } & Nominal & 20.0 & 20.0 & 20.0 & 20.0 & 20.0 & - \\
& DR & 15.5 & 22.7 & 19.5 & 25.7 & 16.6 & - \\
\hline
\end{tabular}

\section{Results}

Figure 1a-f are BSE images of experimental Hf-Mo-Nb-Ta-Ti-Zr alloys. A typical dendritic structure is observed. Their compositions as obtained from SEM-EDS are shown in Table 1. It is noted that the dendritic area is rich in Ta and Mo which have the highest two melting points. This is expected since high melting point elements tend to crystalize first during solidification. By contrast of dendritic structure is poor in HfMoNbTiZr alloy as observed in the BSE image. This means the partition between dendrite and interdendrite is small and the coring phenomenon was less obvious. This is due to the subtraction of the highest melting point element Ta which would solidify first with a Ta-rich solid solution.

Figure 2 shows the X-Ray diffraction patterns. The main phase of the Hf-Mo-Nb-Ta-Ti-Zr alloy series is a BCC disordered solid solution. The asymmetry of (200) and (211) peaks are shown in the diffraction pattern results from the cored dendritic structure. The composition variation of dendritic and interdendritic areas causes a little difference in the lattice constant. The lattice constants of the Hf-Mo-Nb-Ta-Ti-Zr alloy series listed in Table 2 are in the range of 3.305 to $3.400 \AA$ A calculated by the Nelson-Riley extrapolation function. Referring to the phase diagrams of each binary alloy between $\mathrm{Hf}, \mathrm{Mo}, \mathrm{Nb}, \mathrm{Ta}, \mathrm{Ti}$, and $\mathrm{Zr}$, Hf-Mo, and Mo-Zr binary alloys form $\mathrm{Mo}_{2} \mathrm{Hf}$ and $\mathrm{Mo}_{2} \mathrm{Zr}$, respectively, in a certain range of composition even at high temperature. However, these two intermetallic compounds or others do not show up in Hf-Mo-Nb-Ta-Ti-Zr alloys, which means the high entropy effect has a significant benefit in forming simple BCC solid solution in this alloy system especially at high temperature. 


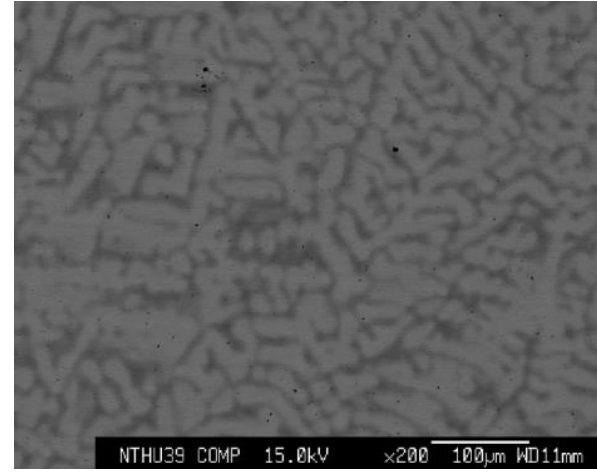

(a)

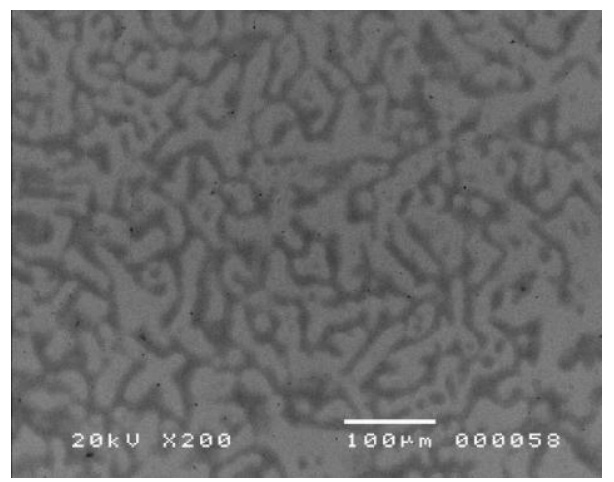

(c)

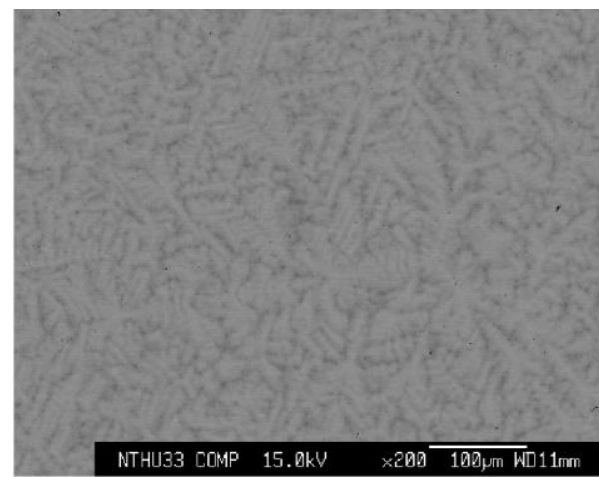

(e)

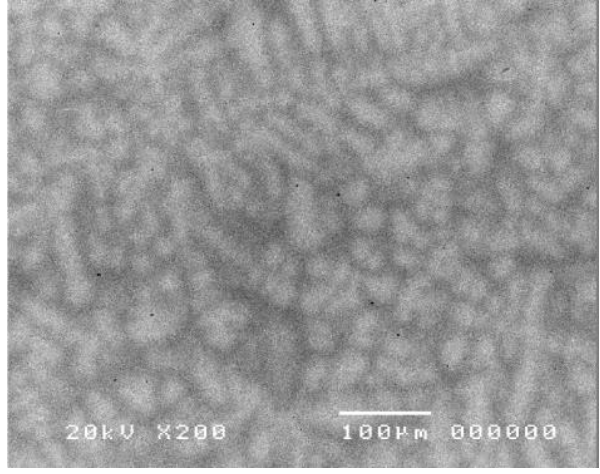

(b)

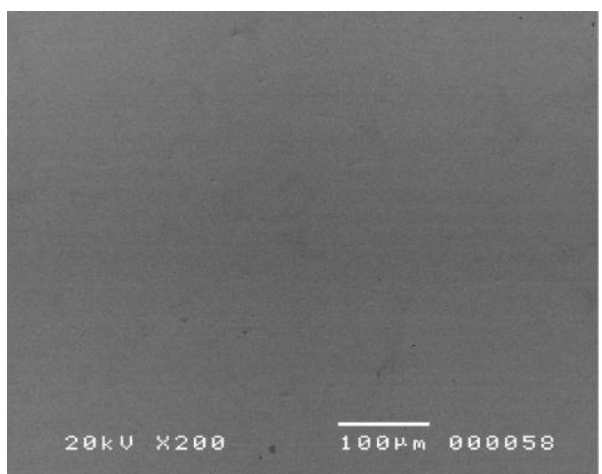

(d)

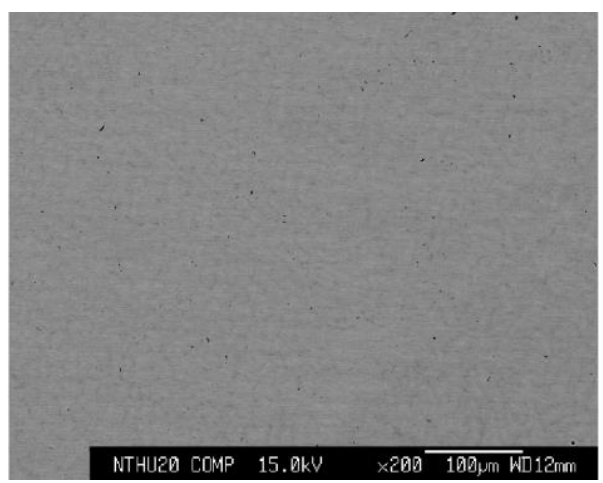

(f)

Figure 1. Backscattering electron (BSE) images of (a) HfMoNbTaTiZr, (b) HfNbTaTiZr, (c) HfMoTaTiZr, (d) HfMoNbTiZr, (e) HfMoNbTaZr, and (f) HfMoNbTaTi. All the alloys show the dendritic structure except HfMoNbTiZr.

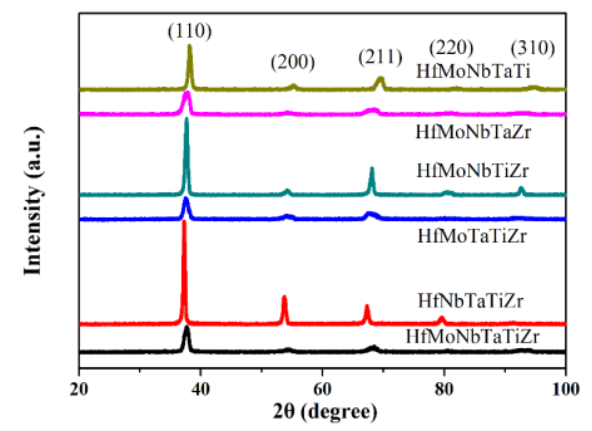

Figure 2. X-ray diffraction patterns of Hf-Mo-Nb-Ta-Ti-Zr alloy series. 
Table 2. The lattice constants ( $\mathrm{\AA})$ of the Hf-Mo-Nb-Ta-Ti-Zr alloy series. Cal. means the value calculated from Vegard's Law. Exp. means the value calculated by Nelson-Riley extrapolation function based on X-ray diffraction pattern.

\begin{tabular}{ccccccc}
\hline & $\begin{array}{c}\text { HfMoNb } \\
\text { TaTiZr }\end{array}$ & $\begin{array}{c}\text { HfNbTa } \\
\text { TiZr }\end{array}$ & $\begin{array}{c}\text { HfMoTa } \\
\text { TiZr }\end{array}$ & $\begin{array}{c}\text { HfMoNb } \\
\text { TiZr }\end{array}$ & $\begin{array}{c}\text { HfMoNb } \\
\text { TaZr }\end{array}$ & $\begin{array}{c}\text { HfMoNb } \\
\text { TaTi }\end{array}$ \\
\hline Cal. & 3.361 & 3.404 & 3.373 & 3.373 & 3.378 & 3.317 \\
Exp. & 3.345 & 3.400 & 3.364 & 3.369 & 3.347 & 3.305 \\
\hline
\end{tabular}

Figure 3 shows the compression test results of $\mathrm{Hf}-\mathrm{Mo}-\mathrm{Nb}-\mathrm{Ta}-\mathrm{Ti}-\mathrm{Zr}$ alloy series. At room temperature, the yield strength of HfMoNbTaTiZr alloy was $1512 \mathrm{MPa}$, and ultimate strength was $1828 \mathrm{MPa}$ when the strain was $11 \%$. The compression tests for HfMoNbTaTiZr alloy were also conducted at $800{ }^{\circ} \mathrm{C}, 1000{ }^{\circ} \mathrm{C}$, and $1200{ }^{\circ} \mathrm{C}$, respectively. At $800{ }^{\circ} \mathrm{C}$, the yield strength of $\mathrm{HfMoNbTaTiZr}$ alloy was $1007 \mathrm{MPa}$ and ultimate strength was $1489 \mathrm{MPa}$ when the strain was $19 \%$, which shows obvious work hardening. At $1000{ }^{\circ} \mathrm{C}$ and $1200{ }^{\circ} \mathrm{C}$, the results of yield strength were $814 \mathrm{MPa}$ and $556 \mathrm{MPa}$, respectively, but the strength kept decreasing from the yield point to the end of the test, showing the work softening behavior. No crack was observed at $1000{ }^{\circ} \mathrm{C}$ and $1200{ }^{\circ} \mathrm{C}$.

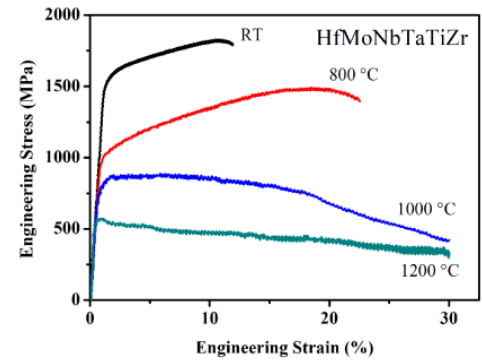

(a)

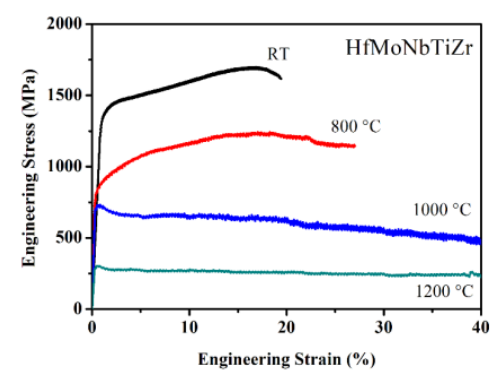

(c)

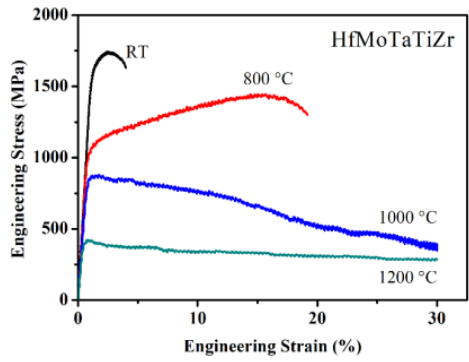

(b)

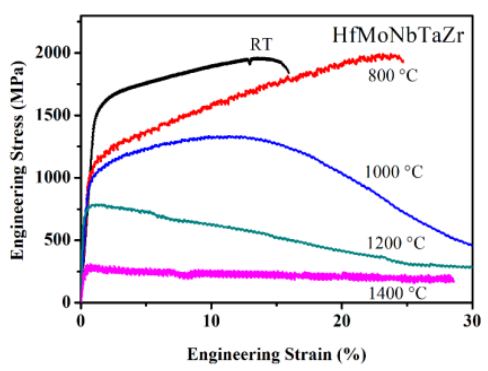

(d)

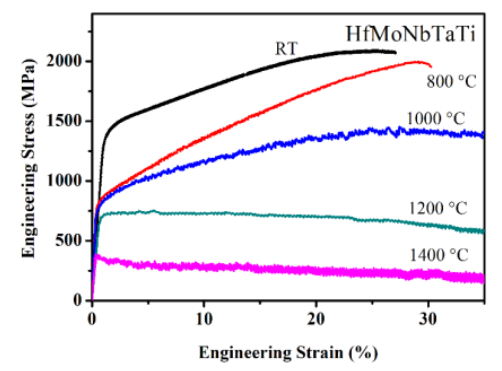

(e)

Figure 3. Engineer compressive stress-strain curve of (a) HfMoNbTaTiZr, (b) HfMoTaTiZr, (c) HfMoNbTiZr, (d) HfMoNbTaZr, and (e) HfMoNbTaTi. 
When an element is removed from HfMoNbTaTiZr, the behavior is changed. The subtraction of $\mathrm{Nb}$ gives HfMoTaTiZr alloy. At room temperature, the yield strength of HfMoTaTiZr alloy was $1600 \mathrm{MPa}$, and the ultimate strength was $1743 \mathrm{MPa}$ when the strain was $3 \%$. At $800{ }^{\circ} \mathrm{C}$, the yield strength was $1045 \mathrm{MPa}$, and the ultimate strength was $1446 \mathrm{MPa}$ when the strain was $23 \%$. As for the HfMoNbTaTiZr alloy, the stress-strain curve of HfMoTaTiZr alloy shows obvious work hardening effect. The results of yield strength were $855 \mathrm{MPa}$ and $404 \mathrm{MPa}$ at $1000{ }^{\circ} \mathrm{C}$ and $1200{ }^{\circ} \mathrm{C}$, respectively. The strength kept decreasing from the yield point to the end of the test. No crack was observed at $1000{ }^{\circ} \mathrm{C}$ and $1200{ }^{\circ} \mathrm{C}$.

The subtraction of Ta from HfMoNbTaTiZr gives HfMoNbTiZr. At room temperature, the yield strength of alloy was $1351 \mathrm{MPa}$, and the ultimate strength was $1698 \mathrm{MPa}$ when the strain was $17 \%$. This alloy performs with better toughness than HfMoNbTaTiZr and HfMoTaTiZr does at room temperature. At $800{ }^{\circ} \mathrm{C}$, the yield strength was $829 \mathrm{MPa}$ and the ultimate strength was $1244 \mathrm{MPa}$ when the strain was $18 \%$. At $1000{ }^{\circ} \mathrm{C}$, yield strength was $721 \mathrm{MPa}$, and the strength kept decreasing when the strain increased. At $1200^{\circ} \mathrm{C}$, the yield strength was $301 \mathrm{MPa}$. The strength of the alloy was almost constant after the yield point to the end of the test. The strain softening effect was balanced by the strengthening effect.

Ti was removed in sequence. At room temperature, the yield strength of HfMoNbTaZr alloy was $1524 \mathrm{MPa}$, and the ultimate compress strength was $1963 \mathrm{MPa}$ when the strain was $13.5 \%$. At $800{ }^{\circ} \mathrm{C}$, the compressive yield strength was $1005 \mathrm{MPa}$, and the ultimate strength was $1991 \mathrm{MPa}$ when the strain was $24 \%$. As with HfMoNbTaTiZr, there is an obvious work hardening effect shown at $800{ }^{\circ} \mathrm{C}$ in the stress-strain diagram. The yield strength was $927 \mathrm{MPa}$ at $1000^{\circ} \mathrm{C}$. There was still a work hardening effect at the beginning of the test. The ultimate strength was $1336 \mathrm{MPa}$ when the strain was $11 \%$, but the strength decreased drastically to $464 \mathrm{MPa}$ at the end of the test. At $1200{ }^{\circ} \mathrm{C}$, the yield strength was $694 \mathrm{MPa}$, but the strength decreased to $289 \mathrm{MPa}$ when the test stopped at $30 \%$ strain. At $1400{ }^{\circ} \mathrm{C}$, the yield strength was $278 \mathrm{MPa}$, and the strength barely decreased during the test. Except for $800{ }^{\circ} \mathrm{C}$, no fracture was observed during the test at elevated temperatures.

Finally, $\mathrm{Zr}$ was removed. At room temperature, the yield strength of HfMoNbTaTi alloy was $1369 \mathrm{MPa}$, and the ultimate compress strength was $2094 \mathrm{MPa}$ when the strain was $25 \%$. The yield strength at $800{ }^{\circ} \mathrm{C}$ was $822 \mathrm{MPa}$, and the ultimate strength was $1998 \mathrm{MPa}$ when the strain was $29 \%$. An obvious work hardening effect can be observed in the stress-strain curve. At $1000{ }^{\circ} \mathrm{C}$, the yield strength was $778 \mathrm{MPa}$, and there was still work hardening effect at the beginning of the test until the ultimate strength $1454 \mathrm{MPa}$, at $27.5 \%$ strain. The results of yield strength were $699 \mathrm{MPa}$ and $367 \mathrm{MPa}$ at $1200{ }^{\circ} \mathrm{C}$ and $1400{ }^{\circ} \mathrm{C}$, respectively. Both stress-strain diagrams show a steady decrease in strength after yield points. No fracture was observed at $1000^{\circ} \mathrm{C}, 1200^{\circ} \mathrm{C}$, and $1400^{\circ} \mathrm{C}$.

The Tables 3 and 4 summarize the results of compressive tests. Comparing the performance at room temperature, HfMoTaTiZr alloy has the best yield strength $1600 \mathrm{MPa}$; HfMoNbTaTi has the highest $27 \%$ fracture strain; comprehensively, HfMoNbTaTi has the best mechanical properties (yield strength $1369 \mathrm{MPa}$ and $27 \%$ fracture strain). The fracture strain increases from $4 \%$ for the HfMoTaTiZr alloy to $12 \%$ for HfMoNbTaTiZr. The presence of Ta can increase the yield strength but decrease the toughness. Ti seems to have no significant influence on strength, but it has a negative effect on toughness. $\mathrm{Zr}$ increases the strength; however, it strongly deteriorates the toughness at room temperature, because the fracture strain decreases from $27 \%$ for the HfMoNbTaTi alloy to $12 \%$ for HfMoNbTaTiZr. The presence of Mo, which has the highest shear modulus, increases the yield strength at room temperature from $929 \mathrm{MPa}$ to $1512 \mathrm{MPa}$ significantly. Nevertheless, the toughness decreases tremendously, fracture strain declines from $>50 \%$ to $12 \%$. However, the presence of $\mathrm{Nb}$ decreases the yield strength only slightly from room temperature to $1000{ }^{\circ} \mathrm{C}$ and increases the yield strength by $38 \%$ at $1200{ }^{\circ} \mathrm{C}$, but largely improves the room temperature fracture strain. This indicates that more $\mathrm{Nb}$ could be added for higher ductility. 
Table 3. The room temperature compressive yield strength and fracture strain of the Hf-Mo-Nb-Ta-Ti-Zr alloy series.

\begin{tabular}{ccccccc}
\hline & HfMoNb & HfNbTa & HfMoTa & HfMoNb & HfMoNb & \multicolumn{2}{c}{ HfMoNb } \\
& TaTiZr & TiZr [25] & TiZr & TiZr & TaZr & TaTi \\
\hline Yield strength $(\mathrm{MPa})$ & 1512 & 929 & 1600 & 1351 & 1524 & 1369 \\
Fracture strain $(\%)$ & 12 & $>50$ & 4 & 20 & 16 & 27 \\
\hline
\end{tabular}

Table 4. The elevated temperature compressive yield strength (MPa) of the Hf-Mo-Nb-Ta-Ti-Zr alloy series.

\begin{tabular}{ccccccc}
\hline \multirow{2}{*}{ Temperature $\left({ }^{\circ} \mathbf{C}\right)$} & $\begin{array}{c}\text { HfMoNb } \\
\text { TaTiZr }\end{array}$ & $\begin{array}{c}\text { HfNbTa } \\
\text { TiZr [25] }\end{array}$ & $\begin{array}{c}\text { HfMoTa } \\
\text { TiZr }\end{array}$ & $\begin{array}{c}\text { HfMoNb } \\
\text { TiZr }\end{array}$ & $\begin{array}{c}\text { HfMoNb } \\
\text { TaZr }\end{array}$ & $\begin{array}{c}\text { HfMoNb } \\
\text { TaTi }\end{array}$ \\
\hline 800 & 1007 & 535 & 1045 & 829 & 1005 & 822 \\
1000 & 814 & 295 & 855 & 721 & 927 & 778 \\
1200 & 556 & 92 & 404 & 301 & 694 & 699 \\
1400 & N. A. & N. A. & N. A. & N. A. & 278 & 367 \\
\hline
\end{tabular}

The melting point of the elements in the alloy affects the strength performance at elevated temperature. For instance, at $1200{ }^{\circ} \mathrm{C}$, HfMoTaTiZr alloy and HfMoNbTiZr alloy which have lower melting-point elements ( $\mathrm{Ta}, \mathrm{Mo}, \mathrm{Nb}$ ) have less strength; HfMoNbTaZr alloy and HfMoNbTaTi alloy which have higher melting-point elements $(\mathrm{Ti}, \mathrm{Zr}$ ) have better strength. Moreover, all the alloys with Mo present have much better strength than HfNbTaTiZr does. Therefore, Mo makes a significant contribution to strength at elevated temperature.

The elevated temperature yield strength versus temperature of Hf-Mo-Nb-Ta-Ti- $\mathrm{Zr}$ alloys is shown in Figure 4a. Except at $800{ }^{\circ} \mathrm{C}$, the strength of the above mentioned Hf-Mo- $\mathrm{Nb}-\mathrm{Ta}-\mathrm{Ti}-\mathrm{Zr}$ alloys is better than the commercial nickel base superalloys, CMSX-4 and Inconel 718. Additionally, Hf-Mo-Nb-Ta-Ti-Zr alloys also have better resistance to softening at elevated temperature. Figure $4 \mathrm{~b}$ is the specific strength of Hf-Mo-Nb-Ta-Ti-Zr alloys, HfNbTaTiZr alloy, CMSX-4, and Inconel 718 at different temperatures. Below $900{ }^{\circ} \mathrm{C}, \mathrm{CMSX}-4$ and Inconel 718 perform better; at $1000{ }^{\circ} \mathrm{C}$, Hf-Mo-Nb-Ta-Ti-Zr alloys, except for HfNbTaTiZr, are better than CMSX-4 and Inconel 718. At temperatures above $1200^{\circ} \mathrm{C}$, HfMoNbTaTi alloy has the highest specific strength.

Comprehensively speaking, Hf-Mo-Nb-Ta-Ti-Zr alloys have a potential application at elevated temperature.

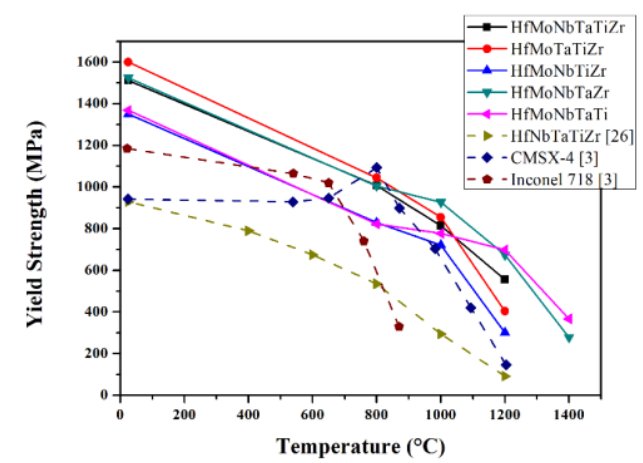

(a)

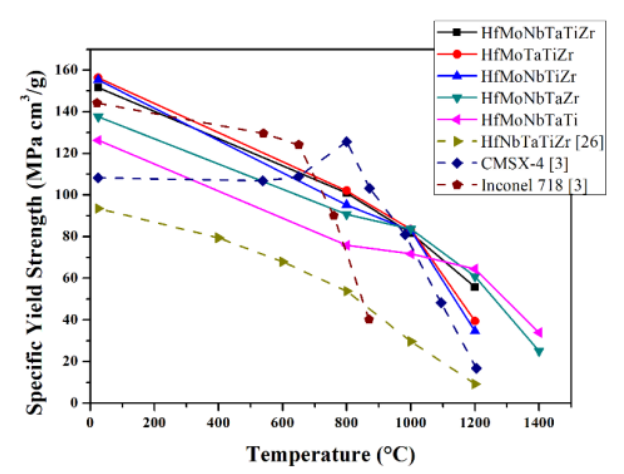

(b)

Figure 4. (a) Elevated temperature yield strength and (b) elevated temperature specific yield strength versus temperature between $\mathrm{Hf}-\mathrm{Mo}-\mathrm{Nb}-\mathrm{Ta}-\mathrm{Ti}-\mathrm{Zr}$ alloy series, CMSC-4, and Inconel 718 [3]. The elevated temperature yield strength of HfNbTaTiZr is from Reference [26]. 


\section{Discussion}

\subsection{Phase Formation Rule}

The solid solution phase formation rules were checked with the microstructure and crystal structure of the alloys in this study. First are the criterion based on thermodynamic parameters and atomic size parameter $[33,34]$. The thermodynamic parameters are mixing entropy $\Delta S_{\text {mix }}$ mixing enthalpy $\Delta H_{m i x}$, and $\Omega$, respectively:

$$
\begin{gathered}
\Delta S_{\text {mix }}=-\sum c_{i} \ln c_{i} \\
\Delta H_{\text {mix }}=\sum 4 \Delta H_{i j} c_{i} c_{j}, i \neq j \\
\Omega=\frac{T_{m} \Delta S_{\text {mix }}}{\Delta H_{\text {mix }}}
\end{gathered}
$$

where $R$ is the gas constant, $c_{i}$ is the atomic percentage of the element $i, c_{j}$ is the atomic percentage of the element $j, \Delta H_{i j}$ is the enthalpy of the binary liquid state of elements $i$ and $j$ at an equiatomic composition from the Miedema's model $[35,36]$, and $T_{m}$ is the melting point of the alloy defined by rule of mixing:

$$
T_{m}=\sum c_{i} T_{m, i}
$$

where $T_{m, i}$ is the melting point of the element $i$. The atomic size parameter is atomic size difference $\delta$ :

$$
\delta=\sqrt{\sum c_{i}\left(1-\frac{r_{i}}{\bar{r}}\right)^{2}}
$$

where $r_{i}$ is the atomic radius of element $i . \bar{r}$ is the average radius of the alloy defined by rule of mixing.

$$
\bar{r}=\sum c_{i} r_{i}
$$

The second criterion determining crystal type is related to electronic parameters, valence electron concentration VEC [37], and the third criterion determining Laves phase is related to Allen electronegativity difference $\Delta \chi_{\text {Allen }}[38]$ :

$$
\begin{gathered}
\text { VEC }=\sum c_{i} V_{E E C_{i}} \\
\Delta \chi_{\text {Allen }}=\sqrt{c_{i}\left(1-\frac{\chi_{i}^{\text {Allen }}}{\bar{\chi}}\right)^{2}}
\end{gathered}
$$

where $V E C_{i}$ is the valence electron concentration of the element $i$ [39], $\chi_{i}^{\text {Allen }}$ is the electronegativity of the element $i$ from Allen et al. [40], and $\bar{\chi}$ is the average electronegativity of the alloy defined by rule of mixing:

$$
\bar{\chi}=\sum c_{i} \chi_{i}^{\text {Allen }}
$$

The ranges in the three criterions for predicting the phases and crystal structure of HEAs are (1) Disorder solid solution phase forms when $\Omega>1.1$ and $\delta<6.6 \%$ [34]; (2) face-centered cubic (FCC) is stable when $V E C>8$, and BCC is stable when $V E C<6.87$ [37]; and (3) Laves phase forms when $\Delta \chi_{\text {Allen }}>7 \%$ and $\delta>5 \%$ in HEAs [41]. All the criterions mentioned above are established through statistical approach, so there is still some error especially in the boundary condition. The properties of pure elements Hf, Mo, Nb, Ta, Ti, and $\mathrm{Zr}$ are listed in Table 5. The BCC atomic radii of $\mathrm{Hf}$, Ti, and $\mathrm{Zr}$ are Goldschmidt radii since all the alloys are a BCC structure. All the parameters of six alloys are calculated and listed in Table 6. From Table 5, one can observe Hf and Zr possess the largest atomic radius and smallest electronegativity, and Mo possesses the smallest atomic radius and biggest electronegativity. Furthermore, from the experiment results, all the alloys at the as-cast state form 
a single BCC disorder solid solution phase and no Laves phase is observed. This indicates that the formation of a single solid solution phase is consistent with criteria (1) and (2) but not consistent with criterion (3). Base on criterion (3), Laves phase might form in all the alloys except HfNbTaTiZr which is at the margin. It is necessary to check the criterion for Laves phase formation because $\mathrm{Mo}_{2} \mathrm{Hf}$ or $\mathrm{Mo}_{2} \mathrm{Zr}$ might form according to the Hf-Mo or Mo-Zr binary phase diagram. The result shows that criterion 3 is not fulfilled in the present alloy series. The minimum value $7 \%$ seems to be lower. One can observe that the $\Omega$ parameter values of these alloys are much higher than 1.1 and the VEC values are significantly lower than 6.87 . This demonstrates that the high entropy effect is significant in enhancing the formation of a solid solution when mixing enthalpy and strain energy is small.

Table 5. Various data of the properties of $\mathrm{Hf}, \mathrm{Mo}, \mathrm{Nb}, \mathrm{Ta}, \mathrm{Ti}$, and $\mathrm{Zr}$. $\mathrm{HCP}$ means hexagonal close-packing.

\begin{tabular}{ccccccc}
\hline $\boldsymbol{\Delta} \boldsymbol{H}_{i j} \mathbf{( k J / m o l )}$ & $\mathbf{H f}$ & $\mathbf{M o}$ & $\mathbf{N b}$ & $\mathbf{T a}$ & $\mathbf{T i}$ & $\mathbf{Z r}$ \\
\hline $\mathrm{Hf}$ & - & -4 & 4 & 3 & 0 & 0 \\
$\mathrm{Mo}$ & -4 & - & -6 & -5 & -4 & -6 \\
$\mathrm{Nb}$ & 4 & -6 & - & 0 & 2 & 4 \\
$\mathrm{Ta}$ & 3 & -5 & 0 & - & 1 & 3 \\
$\mathrm{Ti}$ & 0 & -4 & 2 & 1 & - & 0 \\
$\mathrm{Zr}$ & 0 & -6 & 4 & 3 & 0 & - \\
$r_{i}(\mathrm{~nm})$ & $0.159(\mathrm{HCP})$ & 0.136 & 0.143 & 0.143 & $0.147(\mathrm{HCP})$ & $0.162(\mathrm{HCP})$ \\
$T_{m, i}(\mathrm{~K})$ & $0.155(\mathrm{BCC})$ & 2896 & 2750 & 3290 & 1941 & $0.157(\mathrm{BCC})$ \\
$\chi_{i}^{\text {Allen }}$ & 2506 & 1.47 & 1.41 & 1.34 & 1.38 & 1.32 \\
$V E C_{i}$ & 1.16 & 6 & 5 & 5 & 4 & 4 \\
$G(\mathrm{GPa})$ & 30 & 120 & 38 & 69 & 44 & 33 \\
\hline
\end{tabular}

Table 6. The values of thermodynamics, atomic size, and electronic parameters of the Hf-Mo-Nb-Ta-Ti-Zr alloy serious.

\begin{tabular}{ccccccc}
\hline & HfMoNb & HfNbTa & HfMoTa & HfMoNb & HfMoNb & HfMoNb \\
& TaTiZr & TiZr & TiZr & TiZr & TaZr & TaTi \\
\hline$\Delta H_{\text {mix }}(\mathrm{kJ})$ & -0.9 & 2.7 & -1.9 & -1.6 & -1.1 & -1.4 \\
$\Delta S_{\text {mix }}(\mathrm{J})$ & 14.9 & 13.4 & 13.4 & 13.4 & 13.4 & 13.4 \\
$T_{m}(\mathrm{~K})$ & 2585.2 & 2523.0 & 2552.2 & 2444.2 & 2714.0 & 2676.6 \\
$\Omega$ & 43.3 & 12.4 & 17.8 & 20.4 & 32.4 & 24.9 \\
$\delta$ & $6.3 \%$ & $5.5 \%$ & $6.7 \%$ & $6.7 \%$ & $6.9 \%$ & $5.4 \%$ \\
$\mathrm{VEC}$ & 4.7 & 4.4 & 4.6 & 4.6 & 4.8 & 4.8 \\
$\Delta \chi_{\text {Allen }}$ & $7.2 \%$ & $6.6 \%$ & $7.6 \%$ & $7.8 \%$ & $7.8 \%$ & $7.8 \%$ \\
\hline
\end{tabular}

\subsection{Solution Hardening Mechanism}

The solid solution strengthening effect is calculated to examine the yield strength of the alloys in this study. From the experiment results, all the alloys possess a single phase of BCC disorder solid solution. It is valuable to use the yield strength of the alloys to check the solution strengthening mechanism. The solution strengthening mechanism of HEAs was proposed by Senkov et al. and then modified by Yao et al. [25,42]. The solution strengthening value $\Delta \sigma_{i}$ contributed by element $i$ is:

$$
\Delta \sigma_{i}=A G f_{i}^{4 / 3} c_{i}^{2 / 3}
$$

where $A$ is a material-dependent dimensionless constant of the order of $0.04, G$ is the shear modulus of the alloy, and $f_{i}$ is the mismatch parameter of element $i$ related to shear modulus and atomic size:

$$
f_{i}=\sqrt{\delta_{G, i}{ }^{2}+\alpha^{2} \delta_{r, i}{ }^{2}}
$$


where $\delta_{G, i}$ and $\delta_{r, i}$ are the modulus mismatch parameter and atomic radius mismatch parameter, respectively as Equations (12) and (13). The value of $\alpha$ depends on the type of dislocation. For mixed dislocation, the value is designated to be nine.

$$
\begin{aligned}
\delta_{G, i} & =\frac{9}{8} \sum c_{j} \delta_{G, i j} \\
\delta_{r, i} & =\frac{9}{8} \sum c_{j} \delta_{r, i j}
\end{aligned}
$$

where $\delta_{G, i j}$ and $\delta_{a, i j}$ are the differences between elements $i$ and $j$ in shear modulus and atomic radius, respectively as Equations (14) and (15). Nine is the number of atoms in the $i$-centered cluster in the BCC lattice, eight is the number of atoms neighboring with the center atom $i$.

$$
\begin{gathered}
\delta_{G, i j}=\frac{2\left(G_{i}-G_{j}\right)}{\left(G_{i}+G_{j}\right)} \\
\delta_{r, i j}=\frac{2\left(r_{i}-r_{j}\right)}{\left(r_{i}+r_{j}\right)}
\end{gathered}
$$

where $G_{i}$ and $G_{j}$ are the shear modulus of element $i$ and $j$, respectively, and $r_{j}$ is the atomic radius of element $j$. Eventually, the solution strengthening $\Delta \sigma$ contributed by all the alloying elements is obtained by summation of $\Delta \sigma_{i}$. The calculated yield stress $\sigma_{c}$ is the summation of the yield stress, $\sigma_{m}$, by rule of mixing and $\Delta \sigma$.

$$
\begin{gathered}
\Delta \sigma=\left(\sum\left(\Delta \sigma_{i}\right)^{3 / 2}\right)^{2 / 3} \\
\sigma_{c}=\sigma_{m}+\Delta \sigma
\end{gathered}
$$

As the shear modulus of the HfMoNbTaTiZr alloy system is still lacking, we reasonably use the rule of mixing to calculate it since the modulus relates to the interatomic potential energy well:

$$
G_{m}=\sum c_{i} G_{i}
$$

The calculated results are listed in Table 7 and compared in Figure 5. One can observe that the $\sigma_{m}$ of all the alloys is small and almost the same. In addition, the trends of $\Delta \sigma$ and $\sigma_{c}$ are consistent with $\sigma_{0.2}$. This means that high yield strength of this alloy series all comes from solution strengthening effect despite there being some deviation, about $30 \%$, between calculated values and experimental values. It is interesting to note that Mo, with the smallest atomic radius and the largest shear modulus, interacts frequently with other elements, thus, the HfNbTaTiZr alloy possesses the smallest yield stress without the addition of Mo. Ti, having the average atomic radius and the average shear modulus, interacts slightly with other elements, and, thus, HfMoNbTaZr alloy possess the largest $\Delta \sigma$ without the addition of Ti. As for the deviation between $\sigma_{c}$ and $\sigma_{0.2}$, it might be due to the overestimated shear modulus. Young's modulus of HfNbTaTiZr is 81 GPa reported by Juan et al. [43], and, thus, the shear modulus can be calculated to be $31 \mathrm{GPa}$. This value is obviously smaller than the average shear modulus $43 \mathrm{GPa}$ of HfNbTaTiZr. This implies that all the average shear moduli might be overestimated. If we calculated the shear modulus from the experimental $\sigma_{0.2}$, by assumption that $\sigma_{c}$ equals to $\sigma_{0.2}$, the result is shown in the $G_{c a l}$ column of Table 7. From the figure, the trend of calculated shear modulus $G_{c a l}$ is consistent with the trend of average shear modulus although significantly smaller than $\mathrm{G}_{m}$ by $\sim 30 \%$. One can observe that the calculated shear modulus of HfNbTaTiZr 32 GPa is in a good agreement with the literature [43]. This indicates that severe lattice distortion in HEAs has a strong solution hardening effect, but Young's modulus was effectively lower. This is reasonable and could be related to its effect on lattice constant [11]. In the alloy series, $\mathrm{NiCo}, \mathrm{NiCoFe}, \mathrm{NiCoFeCr}$, and $\mathrm{NiCoFeCrMn}$, the lattice constant of real crystal structure has more deviation from that predicted by Vegard's law. The increased lattice constants as compared with ideal average lattice constant indicates that lattice distortion has 
the effect to expand the lattice. Thus, the interatomic bonding strength is effectively lower and the shear modulus is simultaneously lower. However, in the present alloy series, the calculated lattice constants based on Vegard's law are larger than the experimental lattice constants measured from X-ray diffraction patterns as listed in Table 2, especially with the addition of Mo. This is because Mo has a strong interaction with other elements to reduce the bond length according to $\Delta H_{i j}$ in Table 5 . $\mathrm{Al}$ has the same effect and is reported in Reference [27]. However, there needs to be more research in the future to confirm the reasons for the reduced shear modulus.

Table 7. Comparisons of $\mathrm{G}_{m}, \Delta \sigma, \sigma_{m}, \sigma_{c}, \sigma_{0.2}$, and $\mathrm{G}_{c a l}$ of the present alloy series.

\begin{tabular}{lcccccc}
\hline & $\boldsymbol{G}_{\boldsymbol{m}} \mathbf{( G P a )}$ & $\boldsymbol{\Delta} \boldsymbol{\sigma} \mathbf{( M P a})$ & $\left.\sigma_{\boldsymbol{m}} \mathbf{( M P a}\right)$ & $\left.\sigma_{\boldsymbol{c}} \mathbf{( M P a}\right)$ & $\sigma_{\mathbf{0 . 2}}(\mathbf{M P a})$ & $\boldsymbol{G}_{\boldsymbol{c a l}}(\mathbf{G P a})$ \\
\hline HfMoNbTaTiZr & 55 & 1669 & 260 & 1929 & 1512 & 41 \\
HfNbTaTiZr & 43 & 938 & 225 & 1163 & $929[26]$ & 32 \\
HfMoTaTiZr & 60 & 1918 & 264 & 2182 & 1600 & 41 \\
HfMoNbTiZr & 53 & 1683 & 278 & 1961 & 1351 & 33 \\
\hline HfMoNbTaZr & 58 & 1948 & 273 & 2221 & 1524 & 37 \\
HfMoNbTaTi & 60 & 1610 & 256 & 1866 & 1369 & 41 \\
\hline
\end{tabular}

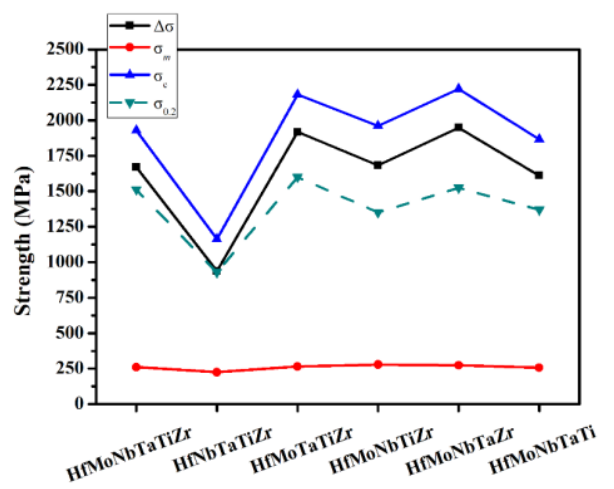

(a)

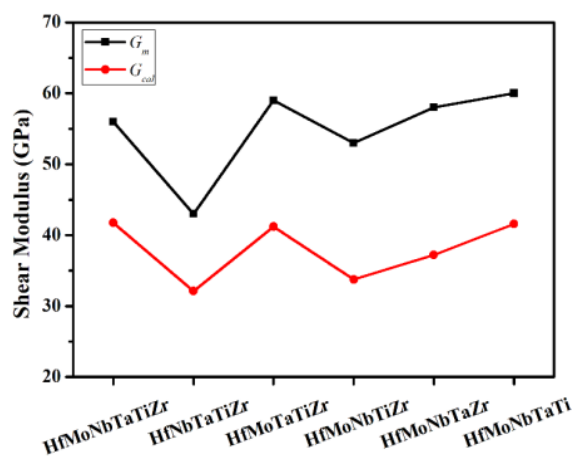

(b)

Figure 5. The trend of Hf-Mo-Nb-Ta-Ti-Zr alloy series: (a) $\Delta \sigma, \sigma_{m}, \sigma_{\mathcal{c}}$, and $\sigma_{0.2}$, and (b) $G_{m}$ and $G_{c a l}$.

\section{Conclusions}

The equiatomic HfMoNbTaTiZr alloy was chosen to analyze the effect of each constituent elemental by the subtraction method and, thus, HfMoNbTaTiZr, HfNbTaTiZr, HfMoTaTiZr, HfMoNbTiZr, HfMoNbTaZr, and HfMoNbTaTi were studied. Among these alloys, HfMoNbTaTi has the best mechanical performance, that is, $27 \%$ compressive strain at room temperature and yield strength $367 \mathrm{MPa}$ at $1400{ }^{\circ} \mathrm{C}$. HfMoNbTaTi has great potential for elevated-temperature applications. As the alloy system does not contain very expensive elements such as $R e$ and $R u$, it is cost competitive for high-temperature applications like $\mathrm{Nb}-\mathrm{Hf}-\mathrm{Ti}$ alloys in space vehicles. Further modification of composition and/or anti-oxidation coatings are still required for high-temperature applications in the air.

According to the experiment results, the effects of $\mathrm{Mo}, \mathrm{Nb}, \mathrm{Ta}$, $\mathrm{Ti}$, and $\mathrm{Zr}$ on mechanical properties of equiatomic Hf-Mo-Nb-Ta-Ti-Zr alloys were described. For higher room-temperature strength, one should add an element which interacts frequently with the alloy, such as Mo. For higher elevated-temperature strength, one should add the elements which possess high melting points, such as $\mathrm{Mo}, \mathrm{Nb}$, or $\mathrm{Ta}$. One should add more $\mathrm{Nb}$ for higher ductility. With $\mathrm{Ti}$ or $\mathrm{Zr}$ addition, the elevated-temperature strength and the density decreases. All these elemental effects could also be applied to all other RHEAs systems, but more research is required to confirm this premise.

The solid solution phase formation rule and the solid solution strengthening effect of RHEAs have been discussed. The high entropy effect of the present alloys is significant in enhancing the formation 
of a solid solution. The shear modulus of RHEAs is smaller than that predicted from mixture rule by about $30 \%$. This reduction is attributable to severe lattice distortion.

Author Contributions: K.-K.T. did the writing-original draft preparation, writing-review \& editing, formal analysis, and the investigation. C.-C.J. did the methodology and the data curation. S.T. and H.-C.C. did the writing-original draft preparation. C.-W.T. and J.-W.Y. did the writing-review \& editing, supervision, project administration, and funding acquisition.

Funding: This work was financially supported by the "High Entropy Materials Center" from the Featured Areas Research Center Program within the framework of the Higher Education Sprout Project by the Ministry of Education (MOE) and from the Project MOST 107-3017-F-007-003 by Ministry of Science and Technology (MOST) in Taiwan.

Acknowledgments: The authors acknowledged Woei-Ren Wang (Industrial Technology Research Institute, Tainan, Taiwan), who provided all the high temperature compressive test with Gleeble 3500.

Conflicts of Interest: The authors declare no conflict of interest.

\section{References}

1. International, A.S.M.; Handbook, C. Properties and Selection: Nonferrous Alloys and Special-Purpose Materials, ASM Handbook, 10th ed.; ASM International: Materials Park, OH, USA, 1990; Volume 2.

2. Smith, W.F. Structure and Properties of Engineering Alloys, 2nd ed.; McGraw-Hill: New York, NY, USA, 1993; 630p.

3. Reed, R.C. The Superalloys: Fundamentals and Applications; Cambridge University Press: Cambridge, UK, 2006. [CrossRef]

4. Kawagishi, K.; Yeh, A.C.; Yokokawa, T.; Kobayashi, T.; Koizumi, Y.; Harada, H. Development of an Oxidation-Resistant High-Strength Sixth-Generation Single-Crystal Superalloy TMS-238. Superalloys 2012. [CrossRef]

5. Satya Prasad, V.V.; Baligidad, R.G.; Gokhale, A.A. Niobium and Other High Temperature Refractory Metals for Aerospace Applications. In Aerospace Materials and Material Technologies: Volume 1: Aerospace Materials; Prasad, N.E., Wanhill, R.J.H., Eds.; Springer: Singapore, 2017; pp. 267-288.

6. Yeh, J.W.; Chen, S.K.; Lin, S.J.; Gan, J.Y.; Chin, T.S.; Shun, T.T.; Tsau, C.H.; Chang, S.Y. Nanostructured high-entropy alloys with multiple principal elements: Novel alloy design concepts and outcomes. Adv. Eng. Mater. 2004, 6, 299-303. [CrossRef]

7. Yeh, J.W. Recent progress in high-entropy alloys. Annales de Chimie Science des Matériaux 2006, 31, $633-648$. [CrossRef]

8. Miracle, D.B.; Senkov, O.N. A critical review of high entropy alloys and related concepts. Acta Mater. 2017, 122, 448-511. [CrossRef]

9. Cheng, C.Y.; Yang, Y.C.; Zhong, Y.Z.; Chen, Y.Y.; Hsu, T.; Yeh, J.W. Physical metallurgy of concentrated solid solutions from low-entropy to high-entropy alloys. Curr. Opin. Solid State Mater. 2017, 21, $299-311$. [CrossRef]

10. Yeh, J.W. Alloy Design Strategies and Future Trends in High-Entropy Alloys. JOM 2013, 65, $1759-1771$. [CrossRef]

11. Gao, M.C.; Yeh, J.W.; Liaw, P.K.; Zhang, Y. High-Entropy Alloys: Fundamentals and Applications, 1st ed.; Springer International Publishing: Cham, Switzerland, 2016.

12. Murty, B.S.; Yeh, J.W.; Ranganathan, S. High Entropy Alloys; Butterworth-Heinemann: Boston, MA, USA, 2014.

13. Tsai, K.Y.; Tsai, M.H.; Yeh, J.W. Sluggish diffusion in Co-Cr-Fe-Mn-Ni high-entropy alloys. Acta Mater. 2013, 61, 4887-4897. [CrossRef]

14. Shen, W.J.; Tsai, M.H.; Tsai, K.Y.; Juan, C.C.; Tsai, C.W.; Yeh, J.W.; Chang, Y.S. Superior Oxidation Resistance of $\left(\mathrm{Al}_{0.34} \mathrm{Cr}_{0.22} \mathrm{Nb}_{0.11} \mathrm{Si}_{0.11} \mathrm{Ti}_{0.22}\right)_{50} \mathrm{~N}_{50}$ High-Entropy Nitride. J. Electrochem. Soc. 2013, 160, C531-C535. [CrossRef]

15. Shi, Y.; Yang, B.; Liaw, P. Corrosion-Resistant High-Entropy Alloys: A Review. Metals 2017, 7, 43. [CrossRef]

16. Chen, P.; Lee, C.; Wang, S.Y.; Seifi, M.; Lewandowski, J.J.; Dahmen, K.A.; Jia, H.; Xie, X.; Chen, B.; Yeh, J.W.; et al. Fatigue behavior of high-entropy alloys: A review. Sci. China Technol. Sci. 2017, 61, 168-178. [CrossRef]

17. Tsao, T.K.; Yeh, A.C.; Kuo, C.M.; Kakehi, K.; Murakami, H.; Yeh, J.W.; Jian, S.R. The High Temperature Tensile and Creep Behaviors of High Entropy Superalloy. Sci. Rep. 2017, 7, 12658. [CrossRef] [PubMed] 
18. Gludovatz, B.; Hohenwarter, A.; Catoor, D.; Chang, E.H.; George, E.P.; Ritchie, R.O. A fracture-resistant high-entropy alloy for cryogenic applications. Science 2014, 345, 1153-1158. [CrossRef] [PubMed]

19. Senkov, O.N.; Miracle, D.B.; Chaput, K.J.; Couzinie, J.-P. Development and exploration of refractory high entropy alloys-A review. J. Mater. Res. 2018, 1-37. [CrossRef]

20. Yeh, A.C.; Tsao, T.K.; Chang, Y.J.; Chang, K.C.; Yeh, J.W.; Chiou, M.S.; Jian, S.R.; Kuo, C.M.; Wang, W.R.; Murakami, H. Developing New Type of High Temperature Alloys-High Entropy Superalloys. Int. J.Metall. Mater. Eng. 2015, 1. [CrossRef]

21. Lu, Y.; Dong, Y.; Guo, S.; Jiang, L.; Kang, H.; Wang, T.; Wen, B.; Wang, Z.; Jie, J.; Cao, Z.; et al. A promising new class of high-temperature alloys: Eutectic high-entropy alloys. Sci. Rep. 2014, 4, 6200. [CrossRef] [PubMed]

22. Kumar, A.; Gupta, M. An Insight into Evolution of Light Weight High Entropy Alloys: A Review. Metals 2016, 6, 199. [CrossRef]

23. Senkov, O.N.; Wilks, G.B.; Miracle, D.B.; Chuang, C.P.; Liaw, P.K. Refractory high-entropy alloys. Intermetallics 2010, 18, 1758-1765. [CrossRef]

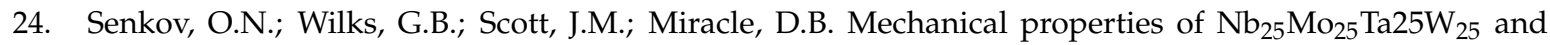
$\mathrm{V}_{20} \mathrm{Nb}_{20} \mathrm{Mo}_{20} \mathrm{Ta}_{20} \mathrm{~W}_{20}$ refractory high entropy alloys. Intermetallics 2011, 19, 698-706. [CrossRef]

25. Senkov, O.N.; Scott, J.M.; Senkova, S.V.; Miracle, D.B.; Woodward, C.F. Microstructure and room temperature properties of a high-entropy TaNbHfZrTi alloy. J. Alloys Compd. 2011, 509, 6043-6048. [CrossRef]

26. Senkov, O.N.; Scott, J.M.; Senkova, S.V.; Meisenkothen, F.; Miracle, D.B.; Woodward, C.F. Microstructure and elevated temperature properties of a refractory TaNbHfZrTi alloy. J. Mater. Sci. 2012, 47, 4062-4074. [CrossRef]

27. Yurchenko, N.; Stepanov, N.; Tikhonovsky, M.; Salishchev, G. Phase Evolution of the AlxNbTiVZr $(x=0 ; 0.5$; 1; 1.5) High Entropy Alloys. Metals 2016, 6, 298. [CrossRef]

28. Lin, C.M.; Juan, C.C.; Chang, C.H.; Tsai, C.W.; Yeh, J.W. Effect of Al addition on mechanical properties and microstructure of refractory $\mathrm{Al}_{x} \mathrm{HfNbTaTiZr}$ alloys. J. Alloys Compd. 2015, 624, 100-107. [CrossRef]

29. Juan, C.C.; Tseng, K.K.; Hsu, W.L.; Tsai, M.H.; Tsai, C.W.; Lin, C.M.; Chen, S.K.; Lin, S.J.; Yeh, J.W. Solution strengthening of ductile refractory $\mathrm{HfMo}_{x} \mathrm{NbTaTiZr}$ high-entropy alloys. Mater. Lett. 2016, 175, $284-287$. [CrossRef]

30. Waseem, O.A.; Lee, J.; Lee, H.M.; Ryu, H.J. The effect of Ti on the sintering and mechanical properties of refractory high-entropy alloy $\mathrm{Ti}_{x} \mathrm{WTaVCr}$ fabricated via spark plasma sintering for fusion plasma-facing materials. Mater. Chem. Phys. 2017. [CrossRef]

31. Wu, Y.D.; Cai, Y.H.; Chen, X.H.; Wang, T.; Si, J.J.; Wang, L.; Wang, Y.D.; Hui, X.D. Phase composition and solid solution strengthening effect in TiZrNbMoV high-entropy alloys. Mater. Des. 2015, 83, 651-660. [CrossRef]

32. Yurchenko, N.Y.; Stepanov, N.D.; Zherebtsov, S.V.; Tikhonovsky, M.A.; Salishchev, G.A. Structure and mechanical properties of B2 ordered refractory AlNbTiVZrx $(x=0-1.5)$ high-entropy alloys. Mater. Sci. Eng. A 2017, 704, 82-90. [CrossRef]

33. Zhang, Y.; Zhou, Y.J.; Lin, J.P.; Chen, G.L.; Liaw, P.K. Solid-Solution Phase Formation Rules for Multi-Component Alloys. Adv. Eng. Mater. 2008, 10, 534-538. [CrossRef]

34. Yang, X.; Zhang, Y. Prediction of high-entropy stabilized solid-solution in multi-component alloys. Mater. Chem. Phys. 2012, 132, 233-238. [CrossRef]

35. Boer, F.R.d. Cohesion in Metals: Transition Metal Alloys; North-Holland: Amsterdam, The Netherlands, 1988; 758p.

36. Takeuchi, A.; Inoue, A. Classification of bulk metallic glasses by atomic size difference, heat of mixing and period of constituent elements and its application to characterization of the main alloying element. Mater. Trans. 2005, 46, 2817-2829. [CrossRef]

37. Guo, S.; Ng, C.; Lu, J.; Liu, C.T. Effect of valence electron concentration on stability of fcc or bcc phase in high entropy alloys. J. Appl. Phys. 2011, 109, 103505. [CrossRef]

38. Poletti, M.G.; Battezzati, L. Electronic and thermodynamic criteria for the occurrence of high entropy alloys in metallic systems. Acta Mater. 2014, 75, 297-306. [CrossRef]

39. Guo, S.; Liu, C.T. Phase stability in high entropy alloys: Formation of solid-solution phase or amorphous phase. Prog. Nat. Sci: Mater. Int. 2011, 21, 433-446. [CrossRef]

40. Mann, J.B.; Meek, T.L.; Knight, E.T.; Capitani, J.F.; Allen, L.C. Configuration Energies of the d-Block Elements. J. Am. Chem. Soc. 2000, 122, 5132-5137. [CrossRef] 
41. Yurchenko, N.; Stepanov, N.; Salishchev, G. Laves-phase formation criterion for high-entropy alloys. Mater. Sci. Technol. 2016, 33, 17-22. [CrossRef]

42. Yao, H.W.; Qiao, J.W.; Hawk, J.A.; Zhou, H.F.; Chen, M.W.; Gao, M.C. Mechanical properties of refractory high-entropy alloys: Experiments and modeling. J. Alloy. Compd. 2017, 696, 1139-1150. [CrossRef]

43. Juan, C.C.; Tsai, M.H.; Tsai, C.W.; Hsu, W.L.; Lin, C.M.; Chen, S.K.; Lin, S.J.; Yeh, J.W. Simultaneously increasing the strength and ductility of a refractory high-entropy alloy via grain refining. Mater. Lett. 2016, 184, 200-203. [CrossRef]

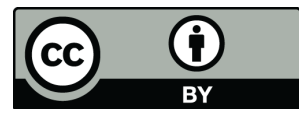

(C) 2018 by the authors. Licensee MDPI, Basel, Switzerland. This article is an open access article distributed under the terms and conditions of the Creative Commons Attribution (CC BY) license (http://creativecommons.org/licenses/by/4.0/). 